\title{
Geogenomic Segregation and Temporal Trends of Human Pathogenic Escherichia coli 0157:H7, Washington, USA, 2005-2014'
}

\author{
Gillian A.M. Tarr, Smriti Shringi, Amanda I. Phipps, Thomas E. Besser, Jonathan Mayer, \\ Hanna N. Oltean, Jon Wakefield, Phillip I. Tarr, Peter Rabinowitz
}

The often-noted and persistent increased incidence of Escherichia coli O157: $\mathrm{H} 7$ infections in rural areas is not well understood. We used a cohort of $E$. coli O157:H7 cases reported in Washington, USA, during 2005-2014, along with phylogenomic characterization of the infecting isolates, to identify geographic segregation of and temporal trends in specific phylogenetic lineages of E. coli O157:H7. Kernel estimation and generalized additive models demonstrated that pathogen lineages were spatially segregated during the period of analysis and identified a focus of segregation spanning multiple, predominantly rural, counties for each of the main clinical lineages, Ib, Ila, and Ilb. These results suggest the existence of local reservoirs from which humans are infected. We also noted a secular increase in the proportion of lineage IIa and IIb isolates. Spatial segregation by phylogenetic lineage offers the potential to identify local reservoirs and intervene to prevent continued transmission.

E scherichia coli $\mathrm{O} 157: \mathrm{H} 7$ infections cause major public health challenges. Most E. coli O157:H7 infections occur sporadically, and the source of infection is often difficult to identify with certainty $(1,2)$. Many reported infections are attributed to food vehicles (1), but studies have implicated other risk factors, and environmental transmission may be particularly notable in rural areas (3-7). Overall, the frequency of infections with $E$. coli $\mathrm{O} 157: \mathrm{H} 7$ has fallen in the United States, which is likely related to improved food safety (8), but it is not clear that rural incidence has also fallen.

Residing in a rural area confers increased risk for $E$. coli $\mathrm{O} 157: \mathrm{H} 7$ infection $(9,10)$. E. coli $\mathrm{O} 157: \mathrm{H} 7$ can persist

Author affiliations: University of Calgary, Calgary, Alberta, Canada (G.A.M. Tarr); Washington State University, Pullman, Washington, USA (S. Shringi, T.E. Besser); University of Washington,

Seattle, Washington, USA (A.I. Phipps, J. Mayer, J. Wakefield,

P. Rabinowitz); Washington State Department of Health,

Shoreline, Washington, USA (H.N. Oltean); Washington University

School of Medicine, St. Louis, Missouri, USA (P.I. Tarr)

DOI: https://doi.org/10.3201/eid2401.170851 in certain locales, posing ongoing risk to humans. Multiple studies demonstrate that specific strains persist within cattle farms and spread to neighboring farms (11-15). The reservoirs enabling this persistence may include water, soil, and wild birds $(16-19)$. It is, therefore, possible that humans incidentally acquire $E$. coli $\mathrm{O} 157: \mathrm{H} 7$ infections because they reside in a geographic region with a persistent reservoir. Using a generalizable population-based cohort, we sought to test the hypothesis that there are geographic foci of related E. coli $\mathrm{O} 157: \mathrm{H} 7$ infections, most likely of environmental origin, taking into account the genomic relatedness of different isolates $(20,21)$ and the geographic, temporal, and secular attributes of their corresponding infections.

\section{Methods}

\section{Study Population and Pathogen Characterization}

We conducted a population-based retrospective cohort study of all culture-confirmed E. coli O157:H7 cases reported to the Washington State Department of Health (DOH; Shoreline, WA, USA) during 2005-2014. E. coli O157:H7 case reporting mandated by the Washington Administrative Code occurs primarily through diagnostic laboratories and healthcare providers. Local health jurisdictions use a standardized $\mathrm{DOH}$ case report form to abstract medical records; interview case-patients to obtain demographic information (including residence address), potential exposures, and details of the course of illness; and determine the most likely source of infection. For this study, case addresses were geocoded and census block groups determined. Case data were deidentified for analysis. This study was deemed exempt by the Washington State Institutional Review Board.

All E. coli $\mathrm{O} 157: \mathrm{H} 7$ isolates are sent to DOH for microbiologic confirmation and XbaI pulsed-field gel electrophoresis (PFGE) typing. We obtained isolates from

${ }^{1}$ Preliminary results from this study were presented at the International Meeting on Emerging Diseases and Surveillance (IMED), November 4-7, 2016, Vienna, Austria. 
$\mathrm{DOH}$ and determined their lineage according to the phylogenetic tree developed by Bono et al. (20) and expanded by Jung et al., who identified some lineages as clinical and others as bovine-biased (21). We used the Jung et al. 48-plex single-nucleotide polymorphism (SNP) assay to type a subset of isolates (21). We assumed that all isolates with a given PFGE pattern would be SNP typed to the same lineage. Thus, we typed $\geq 1$ isolate from each PFGE pattern in the dataset and inferred the lineage of nontyped isolates. Concordance among isolates with identical PFGE profiles was confirmed (online Technical Appendix, https://wwwnc.cdc.gov/EID/article/24/1/170851-Techapp1.pdf). We analyzed the clinically common lineages Ib, IIa, and IIb separately and analyzed the bovine-biased and remaining sparsely represented lineages (21) as a clinically rare group.

\section{Phylogenetic Lineage Spatial Segregation}

Spatial segregation is the ecologic concept that one species or species type is more likely to be surrounded by like than by nonlike individuals (22). We used Diggle's kernel estimation method (23) and spatialkernel package (24) in R (25) to test spatial segregation of E. coli O157:H7 by phylogenetic lineage (online Technical Appendix). In brief, we first estimated a smoothed probability surface for each lineage by comparing the distance between cases infected with the same lineage to the distance between cases infected with different lineages. A peak in the lineage-specific probability surface indicates an area with a high probability of that lineage, relative to the distribution of the other lineages. For example, if $80 \%$ of cases in a given proximity are infected with lineage $\mathrm{Ib}$ but in all other areas lineage $\mathrm{Ib}$ causes only $50 \%$ of cases, we would observe a peak in the lineage Ib-specific probability surface, suggesting spatial segregation. To determine overall spatial segregation, the probability surfaces were compared with a null distribution in which the proportion of infections caused by each lineage is constant across space.

We next sought to account for potential confounders and to detect geographic trends. To do so, we modeled the risk surface using a multinomial generalized additive model (GAM). We estimated the effect of a bivariate thin plate regression spline smooth of latitude and longitude on the odds of infection with a given lineage compared with the most common lineage. This smoothing technique produces a risk surface that can vary flexibly across both horizontal and vertical coordinates. In this analysis, we compared lineages IIa and IIb and the group of clinically rare lineages separately with lineage $\mathrm{Ib}$, which served as the reference (most common) lineage. The model was adjusted for sex and age group $(<5,5-9,10-19,20-59$, and $\geq 60$ years); isolates from cases of unknown age $(\mathrm{n}=1)$ or sex $(\mathrm{n}=$ 10) were excluded from analysis. We estimated parameters using restricted maximum likelihood and the mgcv package in $\mathrm{R}(26,27)$. We further conducted a series of sensitivity analyses to determine the robustness of our results by seeking to confirm our results with 2 independent methods: Dixon's nearest-neighbor test (22) and multinomial spatial scan statistics (28) (online Technical Appendix).

\section{Temporal Variation in Spatial Segregation}

To determine whether spatial segregation of lineages varied over time, we replicated our spatial segregation analyses incorporating time. To do so, we split the years of analysis into 3 intervals (2005-2007, 2008-2010, and 2011-2014) and calculated a kernel-based estimate of spatial segregation for each. We evaluated the effect of time in the multinomial GAM by adding year to the model as a continuous variable, testing the effect of year as both a linear term and as a smoothed term using a thin plate spline. The thin plate spline allows the association between lineage and year to smoothly change in magnitude and direction.

\section{Exploratory Risk Factor Analysis}

We explored potential drivers of segregation by testing the association of risk factors included on the $\mathrm{DOH}$ case report form with each lineage compared with the reference lineage Ib. Using multinomial GAMs adjusting for sex, age, year, and latitude and longitude as a thin plate spline bivariate smoother, we tested each risk factor (online Technical Appendix Table 1). In addition to the statewide analyses, region-specific analyses were conducted for the 3 regions with the highest $E$. coli $\mathrm{O} 157: \mathrm{H} 7$ incidence to determine locally key associations. Regions were defined based on major population centers, areas of increased agricultural intensity, and observed segregation clusters, and models were adjusted for sex, age, and year.

\section{Results}

During the study period, 1,160 E. coli $\mathrm{O} 157: \mathrm{H} 7$ cases were reported to DOH. Of these, 33 isolates, representing 31 PFGE types, were not available for typing (online Technical Appendix), and isolates from 6 cases were excluded as biochemically atypical E. coli $\mathrm{O} 157: \mathrm{H} 7$ (online Technical Appendix Figure 1). We SNP typed 793 isolates and, by extension, matched another 328 to a known lineage using PFGE, enabling us to assign a specific lineage of E. coli $\mathrm{O} 157: \mathrm{H} 7$ to isolates from 1,121 cases. Ten cases lacked address data and were excluded, leaving 1,111 cases for analysis.

Lineages $\mathrm{Ib}$, IIa, and IIb, in descending order, were the most common lineages (Table). Twelve clinically rare lineages were identified, including 2 not previously described, encompassing 45 unique PFGE types (online Technical Appendix Figure 1). Lineage Ib comprised 210 PFGE types, whereas lineage IIa comprised only 38 PFGE types 
Table. Escherichia coli O157:H7 lineage frequency by case characteristic among culture-confirmed human cases reported in Washington, USA, 2005-2014*

\begin{tabular}{|c|c|c|c|c|}
\hline Variable & Lineage $\mathrm{lb}$ & Lineage Ila & Lineage Ilb & Rare lineagest \\
\hline Total & $586(52.7)$ & $260(23.4)$ & $199(17.9)$ & $66(5.9)$ \\
\hline Mean isolates per PFGE type (SD) $\ddagger$ & $2.8(5.3)$ & $6.8(14.3)$ & $7.7(24.7)$ & $1.5(1.7)$ \\
\hline \multicolumn{5}{|l|}{ Sex } \\
\hline $\mathrm{F}$ & $333(56.8)$ & $163(62.7)$ & $105(52.8)$ & $33(50.0)$ \\
\hline M & $244(41.6)$ & $97(37.3)$ & $94(47.2)$ & $32(48.5)$ \\
\hline Unknown & $9(1.5)$ & 0 & 0 & $1(1.5)$ \\
\hline \multicolumn{5}{|l|}{ Age group, y } \\
\hline$<5$ & $119(20.3)$ & $72(27.7)$ & $63(31.7)$ & $10(15.2)$ \\
\hline $5-9$ & $81(13.8)$ & $32(12.3)$ & $33(16.6)$ & $12(18.2)$ \\
\hline $10-19$ & $97(16.6)$ & $51(19.6)$ & $31(15.6)$ & $6(9.1)$ \\
\hline $20-59$ & 207 (35.3) & $81(31.2)$ & 49 (24.6) & $29(43.9)$ \\
\hline$\geq 60$ & $81(13.8)$ & $24(9.2)$ & 23 (11.6) & $9(13.6)$ \\
\hline Unknown & $1(0.2)$ & 0 & 0 & 0 \\
\hline \multicolumn{5}{|l|}{ HUS } \\
\hline Yes & $37(6.3)$ & $18(6.9)$ & $20(10.0)$ & 0 \\
\hline No & 526 (89.2) & $236(90.1)$ & $173(86.1)$ & 67 (98.5) \\
\hline Unknown & $27(4.6)$ & $8(3.1)$ & $8(4.0)$ & $1(1.5)$ \\
\hline
\end{tabular}

and lineage IIb 26 PFGE types (online Technical Appendix Figure 1). Lineage IIa contained an average of 7 (SD 14) and IIb an average of 8 (SD 25) isolates per PFGE type, compared with 3 (SD 5) for lineage Ib and 1 (SD 2) for the clinically rare lineages (Table).

Distribution of cases by sex, age group, and hemolytic uremic syndrome (HUS) status varied by lineage (Table). Lineage IIa and IIb isolates originated disproportionately from children $<5$ years of age compared with isolates in lineage Ib. Patients infected with lineage IIb bacteria also had higher frequencies of HUS (10\%) than other patients $(6 \%)$. None of the patients with infections caused by isolates from the clinically rare lineages developed HUS.

\section{Spatial Segregation}

The result of Diggle's kernel estimation test was statistically significant $(\mathrm{p}=0.001)$, suggesting spatial segregation. Lineage-specific probability surfaces showed separate, distinct peaks for lineages Ib, IIa, and IIb (Figure 1). The southwest region of Washington was marked by segregation of lineage IIb isolates and correspondingly lower probability of isolating lineage Ib from cases. Spatial segregation was observed for lineage $\mathrm{Ib}$ isolates in northwest Washington and for lineage IIa isolates in the south-central region. There was low probability of lineage IIb isolates in both these areas. Sensitivity analysis corroborated these results (online Technical Appendix).

Consistent with the kernel regression results, the adjusted GAM risk surface of lineage IIb varied significantly from that of $\mathrm{Ib}(\mathrm{p}<0.001)$, providing additional support of the spatial segregation. The frequency of lineage IIb isolation was greater than the frequency of Ib in the southwest region, but this imbalance diminished as latitude and longitude increased (Figure 2), that is, in areas northward and eastward. This spatial pattern was also observed in the kernel estimation map of lineage IIb (Figure 1). The risk surfaces of lineage IIa and the clinically rare lineage group did not differ significantly from that of $\mathrm{Ib}$ (online Technical Appendix Table 2). In sensitivity analyses designed to gauge the robustness of results to model assumptions, the spatial risk surface of lineage IIb consistently varied significantly from the risk surface of lineage $\mathrm{Ib}$ (online Technical Appendix Table 2). The spatial risk surface of lineage IIa also varied significantly from the risk surface of lineage $\mathrm{Ib}$ in some sensitivity analyses, similar to the spatial distribution in the kernel estimation lineage IIa-specific probability surface.

We also found significant differences in lineage by age of infected patients, independent of geography. The likelihood of being an adult (age ranges 20-59 and $\geq 60$ years of age) versus being a toddler ( $<5$ years of age) was lower among IIa-infected patients than among Ib-infected patients (20-59 years odds ratio [OR] $0.65,95 \%$ CI $0.44-0.96 ; \geq 60$ years OR $0.49,95 \%$ CI $0.28-0.85$ ). The odds of being 20 59 years of age versus $<5$ years were also lower among IIb-infected patients than among Ib-infected patients (OR $0.44,95 \%$ CI $0.28-0.69)$. Thus, adults comprised a smaller proportion of patients infected with lineage IIa or IIb $E$. coli $\mathrm{O} 157: \mathrm{H} 7$ than of those infected with lineage Ib. We found no significant differences by sex.

\section{Temporal Variation}

The incidence of E. coli $\mathrm{O} 157: \mathrm{H} 7$ averaged 1.73/100,000 population during the study period. Although incidence fluctuated from a low of 1.37/100,000 population in 2014 to a maximum of $2.28 / 100,000$ population in 2013 , we found no discernible trend in overall incidence. However, 
A

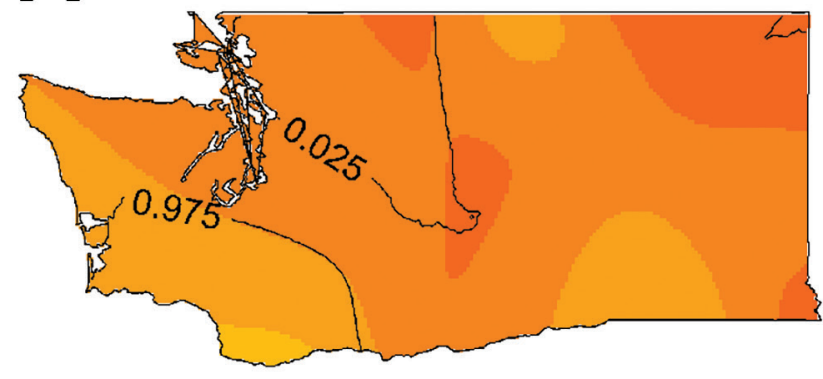

C

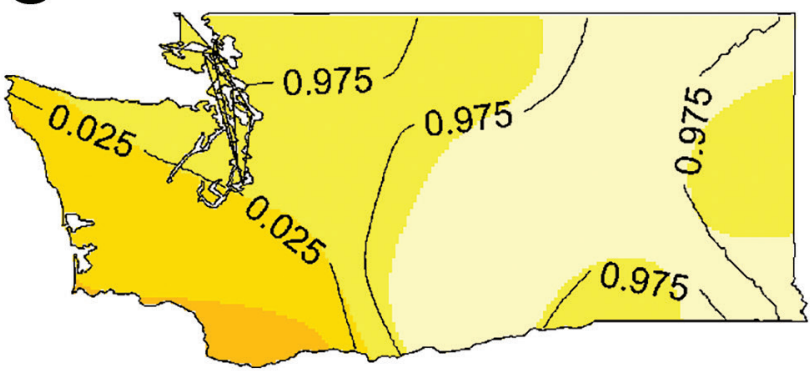

B

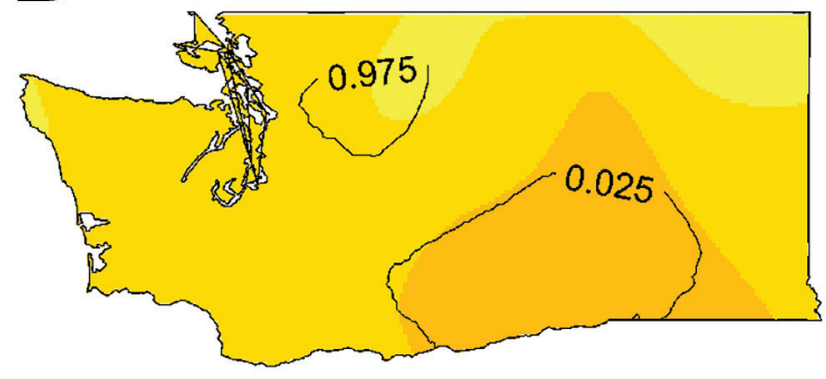

D

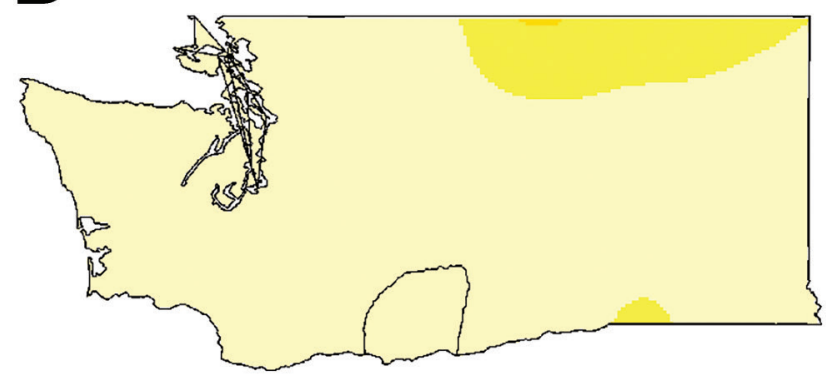

Figure 1. Escherichia coli O157:H7 lineage frequency among culture-confirmed human cases reported in Washington, USA, 2005-2014. A) Lineage Ib; B) lineage Ila; C) lineage Ilb; D) rare lineages (12 different clinically rare lineages). Lineage-specific probability surfaces were determined by kernel-based estimation of spatial segregation. Darker shading indicates higher risk for that lineage. Contour lines marked 0.025 define areas in which there is a high probability of cases being caused by a given lineage, suggesting spatial segregation. Contour lines marked 0.975 define areas in which there is a low probability of cases being caused by the given lineage.

the composition of the E. coli $\mathrm{O} 157: \mathrm{H} 7$ population shifted over time (Figure 3). In the GAM analysis including year as a linear term, incidence relative to lineage $\mathrm{Ib}$ increased over time for lineage IIa (OR 1.26, 95\% CI 1.19-1.34), lineage IIb (OR 1.10, 95\% CI 1.03-1.17), and clinically rare lineages (OR 1.13, 95\% CI 1.02-1.26).

We observed a peak of lineage IIb incidence during the middle of the study period in southwest Washington and the Seattle-Tacoma region (Figure 3). Using kernel regression, we identified statistically significant temporal variation in spatial segregation across intervals $(p=0.001)$. We observed statistically significant overall spatial segregation only during the $2008-2010$ interval $(p=0.001)$. Some portion of the southwest region of the state showed increased probability of lineage IIb isolation during all intervals, and lineages $\mathrm{Ib}$ and IIa were segregated during 2008-2010 and 2011-2014 (Video, https://wwwnc.cdc. gov/EID/article/24/1/17-0851-V1.htm). Cross-validated log-likelihood bandwidths used in these analyses ranged from 0.73 to 1.0. In sensitivity analysis, a lower bandwidth yielded statistically significant spatial segregation during all periods (online Technical Appendix). Latitude and longitude remained significant predictors of $\mathrm{Ib}$ in GAMs that included year (online Technical Appendix Table 2).

\section{Sensitivity Analysis}

Alternate analytic approaches confirmed the results of our primary analyses. Dixon's test for spatial segregation identified statistically significant spatial segregation overall, as well as for lineages Ib, IIa, and IIb (online Technical Appendix Tables 3,4). Three clusters identified using multinomial spatial scan statistics paralleled areas of segregation found in the kernel regression analysis and were consistent with the southwest trend toward proportionally greater IIb observed in the multinomial GAM (online Technical Appendix Figures 3, 4).

To focus on potential local reservoirs, which are not likely to be human, we also conducted the analysis without cases due to presumptive person-to-person transmission (online Technical Appendix). We used the most likely source of infection documented on the DOH case report form to exclude patients most likely infected by other persons. After discounting secondary transmission, we observed spatial segregation using the kernel estimation method $(p=0.002)$. The risk surface of lineage IIb still varied significantly from that of $\mathrm{Ib}(\mathrm{p}<0.001)$. The trend toward greater IIb relative to Ib risk in southwest Washington was consistent with the analysis of all cases, but relative IIb risk was substantially lower in the northeast region than that observed in the primary analysis. 


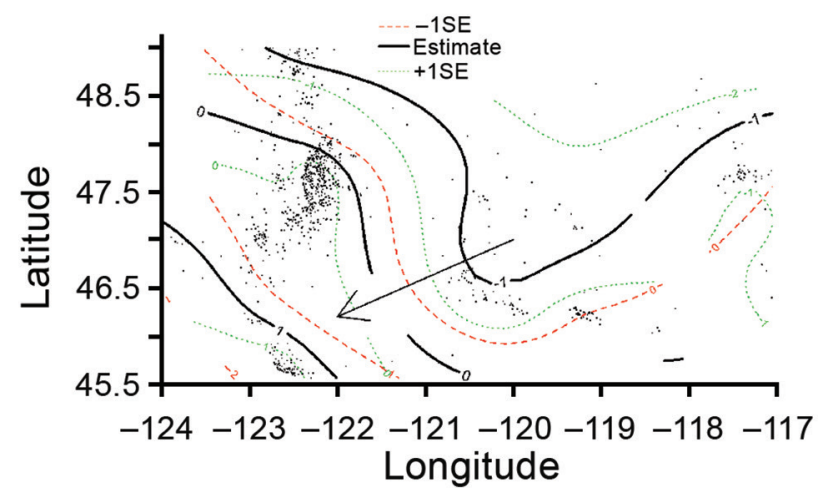

Figure 2. Risk surface of Escherichia coli O157:H7 lineage Ilb relative to lineage $\mathrm{lb}$ using a multinomial generalized additive model and a bivariate thin plate smooth function for longitude and latitude for culture-confirmed human cases reported in Washington, USA, 2005-2014. The black contour lines show the mean effect estimate for lineage $\mathrm{Ilb}$ relative to $\mathrm{lb}$ as latitude and longitude change. The 0 -marked black line indicates no effect. The 1-marked black line indicates greater proportional incidence of lineage Ilb toward the southwest corner of the area as compared to lineage $\mathrm{lb}(\mathrm{p}<0.001)$. The arrow indicates the general direction of the trend from higher Ib risk to higher Ilb risk. Dashed red lines show the effect estimate 1 standard error (SE) below (to the south and west) the mean estimate. Dotted green lines show the effect estimate 1 standard error above (to the north and east) the mean estimate.

This pattern suggests that lineage IIb infections in northeast, but not southwest, Washington may be disproportionately attributed to secondary transmission compared with Ib infections. Finally, we found no evidence of case ascertainment bias that could independently explain our results (online Technical Appendix).

\section{Exploratory Risk Factor Analysis}

Statewide, patients infected with lineage IIa E. coli O157:H7 were more likely to have reported raw fruit or vegetable consumption than those infected with lineage $\mathrm{Ib}$ pathogens (OR 1.81, 95\% CI 1.05-3.11). Patients infected with lineage IIb E. coli $\mathrm{O} 157: \mathrm{H} 7$ were more likely to have reported raw milk consumption than those infected with lineage Ib pathogens (OR 2.46, 95\% CI 1.15-5.28). All examined risk factors and associations are summarized in online Technical Appendix Table 1.

\section{Discussion}

The geographic differences and temporal trends in the relative frequencies of lineages of E. coli $\mathrm{O} 157: \mathrm{H} 7$ from cases in Washington demonstrate that, in addition to genomic variation reported at the national level $(29,30)$, persistent geogenomic variation exists at the regional level. Several geospatial associations warrant elaboration. In all analyses, lineage IIb cases were segregated in the southwest region of the state. Southwest Washington includes Olympia, the state capital, as well as suburbs of Portland, Oregon, north of the Columbia River; however $27 \%$ of the population in the 12 southwest region counties is considered rural, compared with $16 \%$ of the state as a whole (31). Small farms are common. The southwest region is home to $>20 \%$ of the state's farms but accounts for only $7.1 \%$ of its cattle and $6.3 \%$ of farm acreage (32). Roosevelt elk roam the southwest region, and elk elsewhere in the country have been identified as Shiga toxin-producing E. coli carriers (33). Water is also a potential factor in $E$. coli $\mathrm{O} 157: \mathrm{H} 7$ epidemiology in the southwest region, which has abundant coastal and river exposures. The largest recognized IIb outbreak in this region accounted for only 11 cases linked to a particular daycare center (out of $77 \mathrm{IIb}$ infections in the region), so the observed segregation is unlikely due to a single point source. Notably, lineages IIa and IIb have the greatest overlap with the putatively hypervirulent clade 8 (34), making their segregation of particular concern.

Lineage IIb isolates were relatively uncommon in the northwest and south-central regions of Washington, both major cattle-production regions. Lineage Ib showed segregation in the northwest and IIa in the south-central region in some analyses, although their adjusted risk surfaces did not differ significantly, suggesting overlap. More research is needed to clarify why lineage IIb has not yet also established itself in areas with abundant cattle.

The presence of spatially segregated lineages indicates local environmental reservoirs producing infections above and beyond those caused by widely distributed exogenous sources such as food. We propose that persistent spatial segregation of a lineage could reflect a founder effect, in which an ancestral pathogen has become established in a region, persisted, and expanded and occasionally crosses into the human population. Such a dynamic would result in phylogenetically similar bacteria being isolated in the same general geographic region separated by months or years, as we have observed in this study. A possible precedent exists in a report of 2 cases from Webster County, Missouri, USA (35). Our findings are also consistent with those of Jaros et al., who found that geography explains some variation in E. coli $\mathrm{O} 157: \mathrm{H} 7$ strains in New Zealand (36). In addition, prior work from Washington demonstrated shifts over time in the Shiga toxin genotypes of $E$. coli $\mathrm{O} 157: \mathrm{H} 7$ (37).

The clinical infections in our study were dominated by $E$. coli $\mathrm{O} 157: \mathrm{H} 7$ in lineages Ib, IIa, and IIb, consistent with the results of Jung et al. (21). Our work is also consistent with a national study showing that lineage $\mathrm{Ib}$ E. coli O157:H7 causes most clinical cases in the United States (30). Relative to lineage $\mathrm{Ib}$, Washington experienced statistically significant increases in the other clinically common lineages during the study period. The increase is most dramatic for lineage IIa, which appears to have emerged in most regions in the latter half of the study period (Figure 3). 
Figure 3. Annual incidence (per 100,000 population) of reported Escherichia coli O157:H7 cases by phylogenetic lineage, Washington, USA, 2005-2014. A) Statewide; B) northwest region; C) Seattle-Tacoma region; D) southwest region; E) northeast region; F) southcentral region. Regions were defined according to major demographic characteristics and patterns of segregation observed in analyses for the whole period. The northwest region experienced the highest peak incidence. The SeattleTacoma region and the northeast region experienced the lowest incidences. "Rare" indicates 12 different clinically rare lineages. Video. Lineage-specific probability surfaces for Escherichia coli O157:H7 from culture-confirmed human cases reported in Washington, USA, 2005-2014. Probabilities were determined by kernel-based estimation of spatial segregation for 3 intervals: 2005-2007 ( $\mathrm{n}=305$, bandwidth = 1.0000); 2008-2010 ( $\mathrm{n}=367$, bandwidth $=0.7256$ ); and 2011-2014 $(n=439$, bandwidth $=0.9314)$. Overall spatial segregation was not statistically significant for the 2005-2007 interval $(p=0.769)$ or 2011-2014 interval $(p=0.138)$ but was statistically significant for the $2008-2010$ interval $(p=0.001)$. Circles indicate case locations. Darker hues indicate higher risk. Contour lines marked 0.025 define areas in which there is a high probability of cases being caused by a given lineage, suggesting spatial segregation. There is an area of statistically significant spatial segregation for lineage Ilb in all 3 intervals. Contour lines marked 0.975 define areas in which there is a low probability of cases being caused by the given lineage.
This difference could reflect the changing epidemiology of E. coli $\mathrm{O} 157: \mathrm{H} 7$ discussed by Rivas et al., owing to changes in food sources and consumption, or, possibly, pathogen evolution (38). Lineage IIa E. coli O157:H7 has emerged as a major cause of disease across the state, suggesting a disseminated driver of infections for this lineage overall. Lineage IIa's observed association with raw fruit and vegetable consumption, as compared with that for lineage $\mathrm{Ib}$, is consistent with this hypothesis. The south-central region of Washington, identified in some analyses as an area of IIa segregation, experienced an uptick in IIa infections earlier than in other regions. This area includes the Yakima Valley, an area of higher agricultural intensity; a local IIa reservoir in this region could produce the observed segregation independent of statewide trends.

Our findings suggest exposures that may be preferentially associated with particular lineages. Specifically, we observed associations of lineage IIb with drinking untreated/unchlorinated water and raw milk in the southwest region, where this lineage is segregated (online Technical Appendix Table 1). There may be a lineage IIb reservoir in animals producing raw milk in this area, or bacteria from environmental reservoirs in the area may spill over into these animals and local water sources. Only 1 small, recognized raw milk outbreak in 2005 was noted on the DOH case report forms, making it unlikely that a single source is responsible for the association we found over time. It is possible that some $E$. coli $\mathrm{O} 157: \mathrm{H} 7$ lineages may be especially successful in surviving in particular vehicles or environments, such as raw produce or unpasteurized milk or water. Secular changes might also be the result of shifting environmental exposure risk if, for example, contact between a reservoir and humans varies over time. Better knowledge of small-intermediate area transmission patterns will open opportunities for intervention if reservoirs can be identified. 
Our study is limited by its reliance on SNP data to define phylogenetic lineages. Whole-genome sequencing would have supported finer resolution of relatedness, particularly among isolates that were segregated in time and space, and enabled us to trace the history of segregated clusters. Such an analysis would not necessarily alter our conclusions, however, because evolution of specific clades of E. coli $\mathrm{O} 157: \mathrm{H} 7$ within a region, and the identification of different sublineages, would still be consistent with a founder effect. In fact, the precise delineation of the chromosomal architecture in these pathogens might actually confirm a common progenitor, as demonstrated from worldwide analyses of E. coli O157:H7 (39). Our use of phylogenetic lineages rather than PFGE profiles is also a strength of the work, because PFGE does not put differences into evolutionary perspective (39). By basing the analysis on phylogenetic lineages, we captured relatedness among strains and indicate the level of E. coli $\mathrm{O} 157: \mathrm{H} 7 \mathrm{di}-$ versity as it circulates through its host populations. We also used multiple analytic techniques to provide confidence that our results were not due to assumptions made by any particular method.

In summary, clusters of spatial segregation by phylogenetic lineage in Washington suggest local reservoirs that perennially cause human disease. Further exploration of land use, human movements, and social-behavioral factors could elucidate within-region drivers of spatial segregation. We see comparison of lineage-specific spatial patterns with distributions of these and other factors as an essential next step in understanding E. coli $\mathrm{O} 157: \mathrm{H} 7$ spatial segregation. Environmental risk assessment and longitudinal studies based on our findings would also provide valuable information by identifying pathogen reservoirs that have not been identified by traditional public health surveillance and that could be mitigated by public health or environmental measures. The makeup of the E. coli $\mathrm{O} 157: \mathrm{H} 7$ population in the state is also shifting. To manage emerging lineages, attention is needed to the heterogeneity in risk factors across the phylogenetic tree. Greater knowledge of the most likely sources of infection for particular lineages has the potential to focus both outbreak investigations and efforts to identify persistent reservoirs.

This work was supported by the National Institute of Environmental Health Sciences of the National Institutes of Health (award no. T32ES015459) and the National Institute of Allergy and Infectious Disease of the National Institutes of Health (award no. F31AI126834).

Dr. Tarr is a postdoctoral fellow in pediatric enteric infections at the University of Calgary, Calgary, Alberta, Canada. Her primary research interest is the maintenance, distribution, and virulence of zoonotic diseases affecting children.

\section{References}

1. Scallan E, Hoekstra RM, Angulo FJ, Tauxe RV, Widdowson MA, Roy SL, et al. Foodborne illness acquired in the United Statesmajor pathogens. Emerg Infect Dis. 2011;17:7-15. http://dx.doi.org/10.3201/eid1701.P11101

2. Centers for Disease Control and Prevention (CDC). Vital signs: incidence and trends of infection with pathogens transmitted commonly through food-foodborne diseases active surveillance network, 10 U.S. sites, 1996-2010. MMWR Morb Mortal Wkly Rep. 2011;60:749-55.

3. Strachan NJ, Dunn GM, Locking ME, Reid TM, Ogden ID. Escherichia coli O157: burger bug or environmental pathogen? Int J Food Microbiol. 2006;112:129-37. http://dx.doi.org/ 10.1016/j.ijfoodmicro.2006.06.021

4. Denno DM, Keene WE, Hutter CM, Koepsell JK, Patnode M, Flodin-Hursh D, et al. Tri-county comprehensive assessment of risk factors for sporadic reportable bacterial enteric infection in children. J Infect Dis. 2009;199:467-76. http://dx.doi.org/ $10.1086 / 596555$

5. Luffman I, Tran L. Risk factors for E. coli $\mathrm{O} 157$ and cryptosporidiosis infection in individuals in the karst valleys of east Tennessee, USA. Geosciences (Basel). 2014;4:202-18. http://dx.doi.org/10.3390/geosciences4030202

6. Michel P, Wilson JB, Martin SW, Clarke RC, McEwen SA, Gyles CL. Temporal and geographical distributions of reported cases of Escherichia coli O157:H7 infection in Ontario. Epidemiol Infect. 1999;122:193-200. http://dx.doi.org/10.1017/ S0950268899002083

7. Locking ME, O'Brien SJ, Reilly WJ, Wright EM, Campbell DM, Coia JE, et al. Risk factors for sporadic cases of Escherichia coli O157 infection: the importance of contact with animal excreta. Epidemiol Infect. 2001;127:215-20. http://dx.doi.org/10.1017/ S0950268801006045

8. Crim SM, Griffin PM, Tauxe R, Marder EP, Gilliss D, Cronquist AB, et al.; Centers for Disease Control and Prevention (CDC). Preliminary incidence and trends of infection with pathogens transmitted commonly through food-Foodborne Diseases Active Surveillance Network, 10 U.S. sites, 2006-2014. MMWR Morb Mortal Wkly Rep. 2015;64:495-9.

9. Haack JP, Jelacic S, Besser TE, Weinberger E, Kirk DJ, McKee GL, et al. Escherichia coli $\mathrm{O} 157$ exposure in Wyoming and Seattle: serologic evidence of rural risk. Emerg Infect Dis. 2003;9:1226-31. http://dx.doi.org/10.3201/eid0910.020254

10. Innocent GT, Mellor DJ, McEwen SA, Reilly WJ, Smallwood J, Locking ME, et al.; Wellcome Trust-funded IPRAVE Consortium. Spatial and temporal epidemiology of sporadic human cases of Escherichia coli O157 in Scotland, 1996-1999. Epidemiol Infect. 2005;133:1033-41. http://dx.doi.org/10.1017/ S0950268805003687

11. Liebana E, Smith RP, Batchelor M, McLaren I, Cassar C, Clifton-Hadley FA, et al. Persistence of Escherichia coli $\mathrm{O} 157$ isolates on bovine farms in England and Wales. J Clin Microbiol. 2005;43:898-902. http://dx.doi.org/10.1128/ JCM.43.2.898-902.2005

12. LeJeune JT, Besser TE, Rice DH, Berg JL, Stilborn RP, Hancock DD. Longitudinal study of fecal shedding of Escherichia coli $\mathrm{O} 157: \mathrm{H} 7$ in feedlot cattle: predominance and persistence of specific clonal types despite massive cattle population turnover. Appl Environ Microbiol. 2004;70:377-84. http://dx.doi.org/ 10.1128/AEM.70.1.377-384.2004

13. Rosales-Castillo JA, Vázquez-Garcidueñas MS, Alvarez-Hernández H, Chassin-Noria O, Varela-Murillo AI, Zavala-Páramo MG, et al. Genetic diversity and population structure of Escherichia coli from neighboring small-scale dairy farms. J Microbiol. 2011;49:693-702. http://dx.doi.org/10.1007/ s12275-011-0461-2 
14. Herbert LJ, Vali L, Hoyle DV, Innocent G, McKendrick IJ, Pearce MC, et al. E. coli $\mathrm{O} 157$ on Scottish cattle farms: evidence of local spread and persistence using repeat cross-sectional data. BMC Vet Res. 2014;10:95. http://dx.doi.org/10.1186/ 1746-6148-10-95

15. Widgren S, Söderlund R, Eriksson E, Fasth C, Aspan A, Emanuelson U, et al. Longitudinal observational study over 38 months of verotoxigenic Escherichia coli O157:H7 status in 126 cattle herds. Prev Vet Med. 2015;121:343-52. http://dx.doi.org/10.1016/j.prevetmed.2015.08.010

16. Saxena T, Kaushik P, Krishna Mohan M. Prevalence of E. coli O157:H7 in water sources: an overview on associated diseases, outbreaks and detection methods. Diagn Microbiol Infect Dis. 2015;82:249-64. http://dx.doi.org/10.1016/ j.diagmicrobio.2015.03.015

17. Cernicchiaro N, Pearl DL, McEwen SA, Harpster L, Homan HJ, Linz GM, et al. Association of wild bird density and farm management factors with the prevalence of E. coli $\mathrm{O} 157$ in dairy herds in Ohio (2007-2009). Zoonoses Public Health. 2012;59:3209. http://dx.doi.org/10.1111/j.1863-2378.2012.01457.x

18. Barker J, Humphrey TJ, Brown MWR. Survival of Escherichia coli $\mathrm{O} 157$ in a soil protozoan: implications for disease. FEMS Microbiol Lett. 1999;173:291-5. http://dx.doi.org/10.1111/ j.1574-6968.1999.tb13516.x

19. Gargiulo A, Russo TP, Schettini R, Mallardo K, Calabria M, Menna LF, et al. Occurrence of enteropathogenic bacteria in urban pigeons (Columba livia) in Italy. Vector Borne Zoonotic Dis. 2014;14:251-5. http://dx.doi.org/10.1089/vbz.2011.0943

20. Bono JL, Smith TP, Keen JE, Harhay GP, McDaneld TG, Mandrell RE, et al. Phylogeny of Shiga toxin-producing Escherichia coli $\mathrm{O} 157$ isolated from cattle and clinically ill humans. Mol Biol Evol. 2012;29:2047-62. http://dx.doi.org/ $10.1093 / \mathrm{molbev} / \mathrm{mss} 072$

21. Jung WK, Bono JL, Clawson ML, Leopold SR, Shringi S, Besser TE. Lineage and genogroup-defining single nucleotide polymorphisms of Escherichia coli O157:H7. Appl Environ Microbiol. 2013;79:7036-41. http://dx.doi.org/10.1128/ AEM.02173-13

22. Dixon PM. Nearest-neighbor contingency table analysis of spatial segregation for several species. Écoscience. 2002;9:142-51. http://dx.doi.org/10.1080/11956860.2002.11682700

23. Diggle PJ, Zheng P, Durr P. Nonparametric estimation of spatial segregation in a multivariate point process: bovine tuberculosis in Cornwall, UK. Appl Stat. 2005;54:645-58. http://dx.doi.org/ 10.1111/j.1467-9876.2005.05373.x

24. Zheng P, Diggle PJ. Spatialkernel: nonparametric estimation of spatial segregation in a multivariate point process; $\mathrm{R}$ package version 0.4-19. 2013 [cited 2015 May 27] https://CRAN.R-project.org/package=spatialkernel

25. R Core Team. R: A language and environment for statistical computing. Vienna: R Foundation for Statistical Computing; 2015.

26. Wood SN. Fast stable restricted maximum likelihood and marginal likelihood estimation of semiparametric generalized linear models. J R Stat Soc Series B Stat Methodol. 2011;73:3-36. http://dx.doi.org/10.1111/j.1467-9868.2010.00749.x

27. Wood SN. Thin-plate regression splines. J R Stat Soc Series B Stat Methodol. 2003;65:95-114. http://dx.doi.org/10.1111/ 1467-9868.00374
28. Jung I, Kulldorff M, Richard OJ. A spatial scan statistic for multinomial data. Stat Med. 2010;29:1910-8. http://dx.doi.org/ 10.1002/sim.3951

29. Strachan NJ, Rotariu O, Lopes B, MacRae M, Fairley S, Laing C, et al. Whole genome sequencing demonstrates that geographic variation of Escherichia coli O157 genotypes dominates host association. Sci Rep. 2015;5:14145. http://dx.doi.org/10.1038/ srep14145

30. Mellor GE, Fegan N, Gobius KS, Smith HV, Jennison AV, D'Astek BA, et al. Geographically distinct Escherichia coli O157 isolates differ by lineage, Shiga toxin genotype, and total Shiga toxin production. J Clin Microbiol. 2015;53:579-86. http://dx.doi.org/10.1128/JCM.01532-14

31. United States Census Bureau. 2010 Census urban and rural classification and urban area criteria. 2015 [cited 2015 May 27]. https://www.census.gov/geo/reference/ua/ urban-rural-2010.html

32. United States Department of Agriculture. 2012 census of agriculture. Washington: National Agricultural Statistics Service; 2014.

33. Franklin $\mathrm{AB}$, Vercauteren $\mathrm{KC}$, Maguire $\mathrm{H}$, Cichon $\mathrm{MK}$, Fischer JW, Lavelle MJ, et al. Wild ungulates as disseminators of Shiga toxin-producing Escherichia coli in urban areas. PLoS One. 2013;8:e81512. http://dx.doi.org/10.1371/journal.pone.0081512

34. Manning SD, Motiwala AS, Springman AC, Qi W, Lacher DW, Ouellette LM, et al. Variation in virulence among clades of Escherichia coli O157:H7 associated with disease outbreaks. Proc Natl Acad Sci U S A. 2008;105:4868-73. http://dx.doi.org/10.1073/ pnas.0710834105

35. Turabelidze G, Lawrence SJ, Gao H, Sodergren E, Weinstock GM, Abubucker S, et al. Precise dissection of an Escherichia coli O157:H7 outbreak by single nucleotide polymorphism analysis. J Clin Microbiol. 2013;51:3950-4. http://dx.doi.org/10.1128/ JCM.01930-13

36. Jaros P, Cookson AL, Campbell DM, Duncan GE, Prattley D, Carter P, et al. Geographic divergence of bovine and human Shiga toxin-producing Escherichia coli O157:H7 genotypes, New Zealand. Emerg Infect Dis. 2014;20:1980-9. http://dx.doi.org/10.3201/ eid2012.140281

37. Tarr PI, Neill MA, Clausen CR, Newland JW, Neill RJ, Moseley SL. Genotypic variation in pathogenic Escherichia coli O157:H7 isolated from patients in Washington, 1984-1987. J Infect Dis. 1989;159:344-7. http://dx.doi.org/10.1093/infdis/159.2.344

38. Rivas M, Chinen I, Miliwebsky E, Masana M. Risk factors for Shiga toxin-producing Escherichia coli-associated human diseases. Microbiol Spectr. 2014;2. http://dx.doi.org/10.1128/ microbiolspec.EHEC-0002-2013

39. Leopold SR, Magrini V, Holt NJ, Shaikh N, Mardis ER, Cagno J, et al. A precise reconstruction of the emergence and constrained radiations of Escherichia coli $\mathrm{O} 157$ portrayed by backbone concatenomic analysis. Proc Natl Acad Sci U S A. 2009;106:8713-8. http://dx.doi.org/10.1073/ pnas.0812949106

Address for correspondence: Gillian A.M. Tarr, Alberta Children's Hospital, Office C4-634, 2888 Shaganappi Trail NW, Calgary, AB T3B 6A8, Canada; email: gtarr@uw.edu 